\title{
SENECIO INAEQUIDENS DC. (COMPOSITAE, SENECIONEAE), UNA MALEZA PERJUDICIAL INTRODUCIDA EN MÉXICO'
}

\author{
JERZY RZEDOWSKI \\ Instituto de Ecología, A.C., Centro Regional del Bajío \\ Apartado postal 386, 61600 Pátzcuaro, Michoacán \\ HeIKE ViBRANS \\ Especialidad en Botánica, Colegio de Postgraduados \\ 56230 Montecillo, Texcoco, Estado de México \\ Y \\ Graciela Calderón de Rzedowski \\ Instituto de Ecología, A.C., Centro Regional del Bajío \\ Apartado postal 386, 61600 Pátzcuaro, Michoacán
}

\section{RESUMEN}

Senecio inaequidens DC. se registra como maleza invasora de origen sudafricano, presente en al menos una decena de localidades del centro de México. En su mayoría se trata de poblaciones pequeñas de reciente aparición, aunque éste no es el caso de los alrededores de la ciudad de Amealco, ubicada en el sur del estado de Querétaro, donde la planta es sumamente abundante en todo el medio ruderal y al parecer está allí desde hace 50 o más años.

Este artículo resume la información sobre la incertidumbre en la identificación de la especie, en particular en conexión con el uso del nombre Senecio madagascariensis Poir. Incluye asimismo lo más sobresaliente del conocimiento actual acerca de su distribución geográfica, así como de la historia de su proceso invasivo en Europa central y meridional, en Australia y en Argentina, áreas en que la planta se ha convertido en las últimas décadas en maleza altamente perjudicial, sobre todo para los aprovechamientos ganaderos y también como peligroso competidor de especies nativas en algunos ambientes naturales.

Se incluye la descripción e ilustraciones de Senecio inaequidens, así como una discusión de su relevancia ecológica y agronómica.

Palabras clave: Compositae, maleza, México, Senecio inaequidens, Senecio madagascariensis.

${ }^{1}$ Trabajo realizado con apoyo económico del Instituto de Ecología, A.C. (cuenta 902-07), del Consejo Nacional de Ciencia y Tecnología y de la Comisión Nacional para el Conocimiento y Uso de la Biodiversidad. 


\section{ABSTRACT}

Senecio inaequidens DC., a weed of South African origin, is reported from 10 localities of central Mexico. In most places the populations are small and apparently of recent arrival. However, in the vicinity of the town of Amealco, situated in the southern extreme of the state of Querétaro, the species is extremely abundant in ruderal environments and apparently has existed there for 50 or more years.

This paper summarizes information concerning uncertainty of the identification of the species, particularly in connection with the use of the name Senecio madagascariensis Poir. It includes also some outstanding aspects of its geographical distribution and of the history of its invasion in central and southern Europe, Australia and Argentina, where in recent decades the plant has become a severely noxious weed, especially in cattle raising areas, and also a dangerous competitor of native plants in some natural environments.

A description and illustrations of the species are included, as well as a discussion of its ecological and agronomic relevance.

Key words: Compositae, Mexico, Senecio inaequidens, Senecio madagascariensis, weed.

\section{INTRODUCCIÓN}

Bajo el nombre de Senecio burchellii DC. se ha citado recientemente para México (García-Pérez, 2001) una mala hierba ruderal de origen sudafricano. En la presente contribución se ofrece información adicional acerca de esta especie, en parte obtenida de la revisión de la literatura pertinente y en parte procedente de observaciones realizadas en algunas localidades del centro del país.

Se trata de una planta herbácea perenne de vida corta, que puede comportarse como anual (Fig. 1). Florece en el primer año (después de 6 semanas a 3 meses, según la región) y es sensible a heladas fuertes. Es de 15 a $70 \mathrm{~cm}$ de alto; tiene una raíz fibrosa y puede ser leñosa en la porción inferior; generalmente es ramificada desde la base, aunque puede ser más esbelta y con pocas ramas en una población densa. El tallo es estriado y glabro o a veces con algunos pelos esparcidos. Las hojas son alternas, las inferiores a veces dispuestas en fascículos, angostas, sésiles, semiamplexicaules, lineares a oblongo-lanceoladas $u$ oblanceoladas, miden 1 a $4(-8) \mathrm{cm}$ de largo y 1 a $4(-12) \mathrm{mm}$ de ancho, tienen el ápice agudo o acuminado y el margen entero o denticulado, frecuentemente revoluto; rara vez son pinnatipartidas con lóbulos angostos de hasta $5 \mathrm{~mm}$ de largo; son glabras o tienen algunos pelos esparcidos. Las cabezuelas están dispuestas en racimos o cimas corimbiformes, sobre pedicelos cortos o largos (hasta de $13 \mathrm{~cm}$ ), bracteoladas y glabras; el involucro es campanulado a subcilíndrico y consiste de alrededor de 20 brácteas linear-lanceoladas de 3 a $6 \mathrm{~mm}$ de largo y menos de $1 \mathrm{~mm}$ de ancho, así como de un calículo de 8 a 12 bracteolas exteriores lineares e inconspicuas; en fresco las brácteas y bracteolas frecuentemente tienen una mancha oscura en el ápice; las cabezuelas tienen un diámetro de 1.5 a $2 \mathrm{~cm}$ y sus flores son amarillas; presentan (11-)13(-15) flores liguladas con una lámina de 4 a $7(-10) \mathrm{mm}$ de largo y 1 a $2 \mathrm{~mm}$ de ancho, y hasta 100 flores tubulares de 3 a $5 \mathrm{~mm}$ de largo; el receptáculo es plano a convexo y alveolado. Los aquenios son subcilíndricos, de 1.5 a $2.5 \mathrm{~mm}$ de largo, estriados con 9 ó 10 costillas, de color café o negruzco y pubescentes; el vilano consiste de abundantes cerdas de hasta $7 \mathrm{~mm}$ de largo. Se le puede encontrar 
Rzedowski et al.: Senecio inaequidens, una maleza perjudicial introducida en México
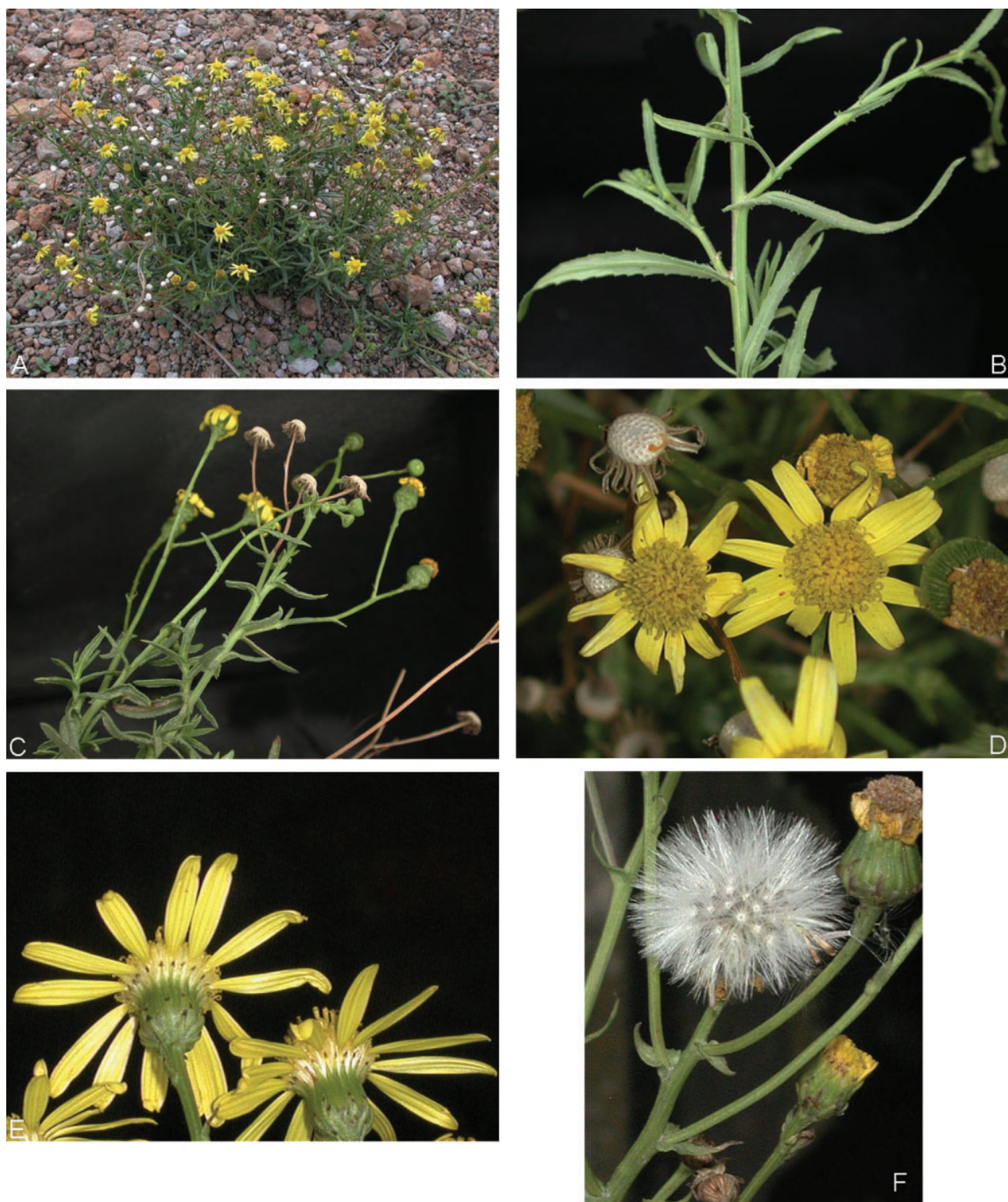

Fig. 1. Senecio inaequidens DC. A. un individuo aislado; B. detalle de hojas superiores; C. inflorescencias; D. cabezuelas vistas de arriba; E. cabezuelas vistas de lado; F. cabezuela madura, mostrando aquenios y vilano. Fotografías de Pedro Tenorio. 
en desarrollo vegetativo y en floración durante prácticamente todo el año, aunque con una notable disminución en diciembre, enero y febrero; en marzo y en abril de nuevo se ven numerosos individuos vigorosos y con muchas cabezuelas, aunque por lo general restringidos a lugares de suelo húmedo. Es planta autógama y produce un alto número de semillas.

La combinación de hojas angostas, semiamplexicaules y casi glabras, las cabezuelas con 13 lígulas, el porte relativamente bajo y la raíz fibrosa, distinguen a este taxon de otras especies de Senecio presentes en el país.

Se conoce en español con los nombres de "botón de oro", "flor amarilla de Mar del Plata" y "senecio amarillo" en Argentina, de "fireweed", "narrow-leaved groundsel" o "variable groundsel" en inglés, de "seneçon de Mazamet" en francés, "senecione sudafricano" en italiano, "schmalblättriges Greiskraut" en alemán.

\section{IDENTIDAD DE LA ESPECIE}

La identificación de esta planta ha tropezado con muchas dificultades y está sin esclarecerse bien todavía, a pesar de ser invasora en varios continentes.

En las floras europeas se le ha denominado por lo general como Senecio inaequidens DC., pero otras veces también como $S$. harveianus MacOwan, S. reclinatus L.f. y S. vimineus Herv. En Australia durante largo tiempo se le consideró como una variante de $S$. lautus Forst.f. ex Willd. en sentido amplio, complejo aún imperfectamente definido de plantas nativas de aquella isla; sólo a partir de 1980 se comprobó que se trata de un elemento exótico y siguiendo la obra de Hilliard (1977) se le determinó como $S$. madagascariensis Poir. En Argentina, en primera instancia se le describió como una especie nueva: $S$. incognitus Cabrera. Posteriormente el mismo Cabrera (Cabrera y Ré, 1965) llegó a la conclusión de que la planta procede de Sudáfrica y tomando en cuenta algunas determinaciones encontradas en herbarios europeos, la identificó tentativamente como $S$. burchellii. En años aún más recientes e indudablemente siguiendo a los autores australianos, se le ha considerado también en ese país sudamericano como $S$. madagascariensis.

En México, su primera colecta de herbario fue inicialmente identificada de manera errónea como S. flaccidus Less. y luego como S. burchellii, basándose en la descripción y en el dibujo de Cabrera (1963). La comparación, sin embargo, de materiales nuestros con otros colectados en Alemania, no revela diferencias significativas y sugiere que ambas pertenecen a la misma especie.

En este mismo contexto cabe señalar que algunos autores consideran que $S$. inaequidens y $S$. madagascariensis constituyen dos taxa distintos, aunque cercanamente emparentados. Tal opinión se ha visto reforzada por el hecho de haber hallado que mientras en las plantas australianas el número cromosómico encontrado es de $2 n=20$, en las europeas ha resultado ser de $2 n=40$. Por otro lado, sin embargo, en el artículo de Scott et al. (1998), basado en el estudio de ADN ribosomal en la región ITS1, se concluye que las poblaciones australianas presentan secuencias idénticas a las de las muestreadas en Sudáfrica e identificadas como $S$. madagascariensis y también a las que se han determinado como $S$. inaequidens de igual procedencia. El mismo trabajo revela pequeñas diferencias entre plantas de $S$. madagascariensis colectadas en Sudáfrica con respecto a las de Madagascar. 
Rzedowski et al.: Senecio inaequidens, una maleza perjudicial introducida en México

En resumen, el estado actual de conocimiento sugiere que el elemento invasor, tanto en Europa, como en Australia y en América, pertenece a una sola especie, de ascendencia sudafricana.

Aun cuando el binomio $S$. madagascariensis tiene prioridad cronológica sobre $S$. inaequidens, es necesaria la realización de un estudio profundo de este difícil complejo sudafricano que incluye también entre otros a $S$. burchellii DC., $S$. harveianus MacOwan, S. pellucidus DC., S. ruderalis Harv. y $S$. skirrhodon DC. De importancia crítica nomenclatural resulta sobre todo la necesidad de definir si la planta invasora es realmente conespecífica con el tipo de $S$. madagascariensis, procedente de una población isleña.

\section{ANTECEDENTES EUROPEOS}

Las siguientes líneas se basan esencialmente en la información resumida en los artículos de Ernst (1998) y de Böhmer (2001), de acuerdo con la cual la planta sudafricana fue introducida en repetidas ocasiones a Europa con la lana de borregos importada para la industria textil. Los primeros registros proceden del norte de Alemania de 1889 y 1896 (puerto de Bremen). Poco después estuvo presente en Francia. En 1922 se le encontró en Bélgica, en 1928 en Escocia, en 1932 con cierta duda en Italia y en 1939 en Holanda.

Durante la primera mitad del siglo XX, sin embargo, sólo parece haber sobrevivido en pequeñas poblaciones situadas en los alrededores de algunas de las fábricas, y no fue sino a partir de 1950 cuando comenzó a expandirse en forma masiva a lo largo del occidente, centro y sur de Europa. Llegó al oeste de Alemania, cerca de Aachen, alrededor de 1970, a Colonia en 1980, en 1985 a la parte central (Hesse) y a la porción sur (Baviera, Stuttgart) de ese país. En la primera mitad de los noventas ya se encontró en Berlín y en Alemania oriental. La expansión principal se llevó a cabo invariablemente a lo largo de vías férreas y autopistas, aunque de allí se ha extendido la planta a otros ambientes ruderales y abiertos. En 1998 se registró del sur de Polonia, el año antepasado se dio a conocer su existencia en la República Checa (Mandak y Bimova, 2001) y en el presente se hizo constar su participación en la vegetación de dunas costeras de las islas Frisias Orientales en el litoral del Mar del Norte (Kuhbier y Weber, 2003).

En 1984 se citó de España y de Austria (Polatschek, 1984); también son varias sus menciones de Inglaterra y de Irlanda.

Los ambientes más comunes habitados por la invasora son las orillas de caminos, de vías férreas y algunos ríos, así como lotes baldíos en áreas industriales, prados y pastizales.

En cuanto a aspectos ecológicos, Ernst (op. cit.) encontró que las plantas son altamente autocompatibles y a principios de verano producen semillas que dan origen a individuos nuevos en el mismo año, mientras que las más escasas semillas que maduran a fines de otoño, presentan un periodo de varios meses de latencia, durante el cual sobreviven en temperaturas menores de $-15^{\circ} \mathrm{C}$.

El mismo autor postuló que la rápida expansión de $S$. inaequidens en Europa, después de un largo periodo de escasa y aislada supervivencia, pudo deberse a la conjunción de cambios ambientales favorables con una selección genética que permitió una mejor adaptación de la planta a condiciones climáticas adversas. 


\section{ANTECEDENTES AUSTRALIANOS}

La información correspondiente se basa en los trabajos de Sindel (1996) y de Radford y Cousens (2000).

De acuerdo con los datos existentes en los herbarios, la planta llegó antes de 1918, posiblemente llevada en lastres de barcos que navegaban de Europa a Australia con escala en los puertos del Cabo Sudafricano.

Se ha establecido a lo largo de la región costera del sureste de la isla, mayormente en el estado de Nueva Gales del Sur (New South Wales), así como en áreas cercanas de Queensland y de Victoria, entre $28^{\circ}$ y $38^{\circ}$ de latitud S.

Prospera principalmente en agostaderos descuidados o de cobertura escasa, así como en los intensamente pastoreados y también en parcelas de cultivo, sobre todo de avena. En la actualidad se considera como una de las malezas que causan mayores estragos en la parte populosa de ese país.

A raíz de tales circunstancias, a partir de 1980 se ha llevado a cabo en Australia un considerable número de estudios tendientes a conocer mejor esta maleza, tanto desde el punto de vista fisiológico-ecológico, de biología reproductiva, de predicción y de control de su expansión, como también de su origen y correcta ubicación taxonómica. Gran parte de esta literatura está inventariada en los trabajos de Sindel (op. cit.), de Scott et al. (op. cit.) y de Radford y Cousens (op. cit.).

\section{ANTECEDENTES ARGENTINOS}

La siguiente información procede mayormente de los artículos de Cabrera y Ré (op. cit.), así como de Verona et al. (1982), de acuerdo con los cuales la especie se registró por primera vez de Argentina en 1940 en el puerto de Bahía Blanca, donde crecía en las calles y en los bordes de caminos aledaños a la ciudad.

Para 1965 ya se había extendido a amplios sectores del este y sureste (en latitudes entre $35^{\circ}$ y $39^{\circ} \mathrm{S}$ ) de la provincia de Buenos Aires, y se encontró asimismo en las de Entre Ríos y Mendoza.

Al igual que en Australia, además de comportarse como ruderal, la planta ha invadido grandes extensiones de agostaderos y de rastrojos de cultivos anuales, interfiriendo en los ciclos de rotación, sobre todo en la fase de la avena.

Además de la problemática ecológica de esta maleza, discutida en el mencionado trabajo de Verona et al., se han realizado en Argentina contribuciones sobre la germinación de sus semillas (Alonso et al., 1982) y sobre sus características reproductivas (Fernández y Verona, 1984).

\section{PRESENCIA EN OTROS PAÍSES}

Como ya se apuntó con anterioridad, todo parece indicar que Senecio inaequidens es nativo de la región costera oriental de África meridional, donde de acuerdo con Hilliard (op. cit.) y Sindel (op. cit.) habita en la provincia de Natal de la Unión Sudafricana y en el sur de Mozambique, en latitudes al sur del paralelo $23^{\circ} 30^{\prime}$. Su real existencia en 
Madagascar está por corroborarse. Cabe mencionar que la especie también está expandiéndose en su región nativa en Sudáfrica, en habitats como orillas de carreteras, superficies quemadas y dunas costeras, habiendo ampliado su área de distribución hasta la costa occidental (Böhmer, 2001).

Sindel (op. cit.) señala en adición que la planta se ha encontrado asimismo en regiones de elevada altitud $(2600-2800 \mathrm{~m})$ de Kenia y de Colombia.

Kinoshita et al. (1999) la citan recientemente de Japón, mientras Motooka et al. (1999) refieren que la especie se observó por primera vez en Hawai antes de 1985 y posteriormente ha estado invadiendo agostaderos en varias de las islas.

\section{PRESENCIA EN MÉXICO}

La primera colecta de Senecio inaequidens de este país procede aparentemente de los alrededores de Amealco, cerca del extremo sur del estado de Querétaro y fue realizada a orillas de camino en 1990.

En 1992 se le localizó en ambientes fuertemente perturbados de San Nicolás Tlaminca, municipio de Texcoco, Estado de México. Desde 1998 se ha encontrado en varias ocasiones en el municipio de Texcoco, generalmente en forma de poblaciones pequeñas o individuos solos. Se colectó en los terrenos del Colegio de Postgraduados en Montecillo y en un terreno baldío en la orilla de la cabecera municipal; se vieron individuos aislados (sin colectar) en el fraccionamiento Lomas de Cristo (2002), y otra vez en Tlaminca, en 2001 y 2002. En 2001 se halló y se muestreó una población grande en las orillas de caminos dominados por Pennisetum clandestinum dentro del poblado Colonia Ávila Camacho, sobre la carretera libre México - Puebla, a los 2910 m s.n.m.

En 1997 se encontró a la orilla de vía de ferrocarril cerca de Puerto Medina, municipio de Contepec, en el extremo nororiental de Michoacán. En 1999 y 2002 se le volvió a muestrear en los alrededores de Amealco, donde se le registró también de áreas inmediatamente aledañas de Michoacán.

En 2000 se localizó la planta en el extremo norte del Estado de México, a lo largo de la carretera México - Querétaro (municipio de Aculco) y en 2001 se ubicó una población pequeña, pero vigorosa, de aproximadamente 20 individuos, en la orilla de la carretera cerca de la ciudad de Toluca.

Las observaciones más metódicas realizadas en los años 2001 y 2002 permitieron apreciar que la gran mayoría de las poblaciones mencionadas son de escasos individuos y sin tendencia visible de rápida expansión.

Una situación muy diferente se registró, sin embargo, en Amealco y áreas cercanas, donde la planta es sumamente abundante y se está comportando como invasora agresiva del medio ruderal, principalmente a lo largo de calles, caminos y veredas, al igual que en lotes baldíos urbanos y suburbanos.

Como dato de particular interés cabe anotar aquí que, si bien en una de las colectas de los alrededores de Amealco se registra el nombre común de "manzanilla de llano", en repetidas encuestas posteriores no se ha logrado confirmar su uso corriente en la región. Todos los informantes de edad coinciden a su vez en afirmar que la planta no es de reciente introducción, sino que la conocen desde que eran niños, o sea que debe tener al menos 50 años de antigüedad en el área. 
En Amealco, la máxima concentración de la especie se observó en las porciones periféricas de la ciudad y con una fuerte expansión a lo largo de las principales carreteras que parten de la población, que son cinco: dos hacia el norte rumbo a San Juan del Río, una hacia el este que conduce a Aculco, una hacia el sur con dirección a Santiago Mezquititlán y Temascalcingo, y una hacia el oeste que lleva a Coroneo y Acámbaro. Un par de estas carreteras fueron construidas y pavimentadas en la década de los cincuentas, siendo sensiblemente posteriores las otras tres y es notable el hecho de que es a lo largo de estas dos vías de comunicación más antiguas, donde se ve mucho más abundante y extendida la maleza a lo largo de varios kilómetros.

Amealco y las áreas inmediatamente aledañas están ubicadas entre 2600 y 2700 m s.n.m. y por extrapolación a partir de localidades vecinas de altitud y condiciones ecológicas similares*, cabe estimarle una precipitación total anual de 700 a $800 \mathrm{~mm}$, mayormente concentrada entre mayo y octubre. La temperatura media anual debe ser del orden de $13^{\circ} \mathrm{C}$, mientras que el promedio de la mínima del mes más frío probablemente se encuentra entre 1 y $2^{\circ} \mathrm{C}$, con la verosímil presencia de 30 a 40 heladas en promedio anual.

De las restantes localidades registradas de los estados de Michoacán y de México unas se hallan en altitudes algo menores y la más baja $(2250 \mathrm{~m})$ corresponde a Montecillo, próximo a Texcoco, donde el promedio anual de la temperatura es de ca. $16^{\circ} \mathrm{C}$ y la precipitación de unos $700 \mathrm{~mm}$. Otras son más altas, como Colonia Ávila Camacho, a casi 3000 m s.n.m., lugar de clima más fresco y con mayor cantidad de lluvia (ca. $11^{\circ} \mathrm{C}$ y ca. $1150 \mathrm{~mm}$ registrados en la cercana estación de Río Frío).

En términos de características morfológicas, las plantas mexicanas coinciden bien con las descripciones y los dibujos de Cabrera (1963), de Pignatti (1982) y de Harden (1992). Solamente en un ejemplar (Vibrans 7389) se le han visto las hojas partidas, como las que registran Harden (1992) para Australia y Cabrera y Ré (op. cit.), así como Verona et. al. (op. cit.) para Argentina.

\section{RELEVANCIA ECOLÓGICA Y AGRONÓMICA}

La información de esta sección se basa principalmente en el trabajo de Böhmer (2001), quien revisa la literatura y resume los resultados de un estudio amplio de la distribución y ecología de la especie en Alemania.

En su área de origen en Sudáfrica Senecio inaequidens crece en laderas inclinadas, dominadas por pastizales, con suelos pedregosos, así como orillas arenosas o guijarreñas de ríos estacionales, entre 1400 y 2850 m s.n.m.

Este tipo de ambiente explica parcialmente sus afinidades ecológicas en otras regiones. Senecio inaequidens inicia sus invasiones habitando lugares abiertos, con suelos sueltos y bien drenados. Se entiende también con facilidad por qué las vías de ferrocarril son tan frecuentemente su puerta de entrada.

Una vez establecida, también se extiende a otros tipos de vegetación que no son estrictamente viarios, ni necesariamente antropogénicos, ni con suelos someros y ligeros.

*Los registros publicados de la estación meteorológica de Amealco no son confiables, pues en primera instancia indican que tal estación está ubicada a $2175 \mathrm{~m}$ de altitud. 
En Argentina y Australia se encuentra como arvense y sobre todo como maleza en agostaderos, potreros (particularmente en los mal cuidados o sobrepastoreados) y cultivos forrajeros. En aquellos países está causando daños cuantiosos (en Australia estimados en alrededor de 2 millones de dólares por año), ya que es tóxica para bovinos, causando malfuncionamiento del hígado parecido a una cirrosis. La toxicidad se debe a alcaloides pirrolizidínicos, los cuales pueden pasar a la leche. Se han registrado intoxicaciones mortales de personas por semillas de Senecio presentes como impurezas en la harina de trigo. El ganado ovino y caprino es más tolerante y solamente exhibe un efecto perjudicial si ingiere grandes cantidades durante un tiempo prolongado.

La planta parece ser capaz de colonizar con éxito en tres tipos principales de ambientes: las porciones subtropicales orientales de los tres continentes del hemisferio sur (Sudáfrica, Australia, Argentina) con indudable influencia marítima; las regiones con clima templado y húmedo, como la de Europa central y occidental (posiblemente auxiliada por una serie de veranos extraordinariamente calurosos en las últimas décadas); así como áreas intertropicales de altitudes elevadas, como es el caso de Colombia, Kenia y México.

Las medidas de combate experimentadas incluyen la aplicación de herbicidas, el impulso de la vitalidad de las plantas cultivadas, ya que la maleza no prospera en la sombra y bajo competencia fuerte, y el pastoreo por ovinos o cabras.

Desafortunadamente, la especie parece ser bastante resistente tanto a varios herbicidas como a cortes mecánicos. Se ha observado una promoción selectiva de Senecio inaequidens con la aplicación de algunos productos químicos y con cortes; tiene buena capacidad de rebrotar aun cuando se siegue a $5 \mathrm{~cm}$ de altura. La medida de control más efectiva consiste en arrancar a mano individuo por individuo, lo que generalmente no es muy difícil por sus raíces superficiales y fibrosas. Es importante no dejar aquenios maduros en el sitio, ya que se ha observado el letargo y la viabilidad del embrión durante muchos años. Las semillas pueden germinar en la oscuridad, pero no emergen de más de $2 \mathrm{~cm}$ de profundidad (Alonso et al., 1982).

En Alemania, la planta se volvió dominante en viñedos abandonados, que anteriormente tenían una vegetación muy diversa, consistente en su gran mayoría de elementos nativos (Fig. 2); también está iniciando la colonización de peñas, habitats rocosos y dunas costeras. En México cabría esperar que pueda invadir pedregales y afloramientos tepetatosos, que a menudo sustentan una flora de especies endémicas y raras. Sobre todo en Australia, ha mostrado una marcada tendencia a colonizar superficies quemadas (de allí su nombre de fireweed en inglés), lo que también puede suceder en México. Con ello la especie puede volverse perjudicial no sólo para la agricultura y la ganadería, sino también para la conservación de la biota nativa.

Es pertinente mencionar que la planta puede prosperar sobre suelos con alto contenido de metales pesados, como lo son los depósitos de desperdicios mineros.

\section{CONSIDERACIONES FINALES}

De toda la información presentada cabe concluir que:

1.- Está aún pendiente la cabal confirmación de que las plantas registradas como invasoras en Argentina, Australia, Europa y México pertenezcan a la misma especie de 


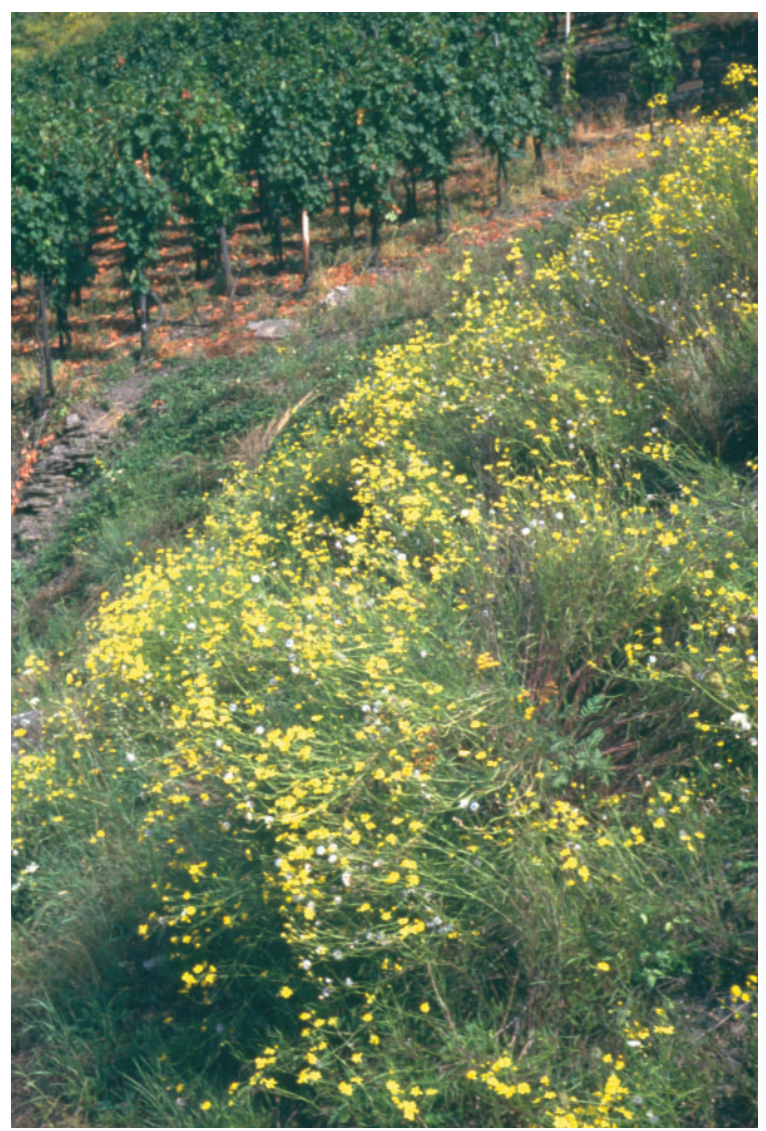

Fig. 2. Invasión de Senecio inaequidens en un viñedo abandonado en el occidente de Alemania.

origen sudafricano. También queda por definir si le corresponde en realidad el nombre de Senecio inaequidens DC.

2.- Se trata de una maleza capaz de ocasionar considerables daños, sobre todo a la ganadería, como ha sucedido en algunas regiones de Australia y de Argentina. También es posible que invada la vegetación nativa, sobre todo la de los riscos y la de los pedregales e inclusive la de los médanos.

3.- Se ha visto que antes de convertirse en mala hierba perjudicial, la especie aparentemente requiere de un prolongado periodo de ajuste genético para adaptarse a las condiciones de su nuevo ambiente. Después es capaz no sólo de colonizar habitats ruderales, sino también lugares cubiertos por vegetación abierta natural o seminatural. 
Rzedowski et al.: Senecio inaequidens, una maleza perjudicial introducida en México

4.- Quedan aún muchas incógnitas en lo que concierne a las tolerancias ecológicas de la planta y su posible valor predictivo. La semejanza del clima de las regiones costeras de Sudáfrica, Argentina y Australia, explica bastante bien el éxito logrado en los últimos dos países. Sin embargo, no sucede lo mismo en el caso de Europa ni tampoco en el de las localidades intertropicales de montaña, donde las condiciones climáticas son muy diferentes, aunque por otro lado es notable la analogía ambiental entre los sitios de México, Colombia y Kenia.

5.- La presencia de la planta en nuestro país quedó inadvertida por muchos años, debido con seguridad a su distribución muy localizada y la poca atención prestada generalmente a la vegetación secundaria. No se sabe como llegó a México ni se entiende por qué se instaló precisamente en Amealco y sus alrededores. No deja de ser significativo su aparentemente reciente surgimiento en el municipio de Texcoco, así como su localización a la orilla de una vía férrea en el este de Michoacán, sobre todo tomando en cuenta el hecho de que Amealco no está comunicado por ferrocarril. Cabe en tal circunstancia la sospecha de que existen en la parte central de la República otro u otros lugares en que está establecida la especie y que funcionan como núcleos de dispersión.

\section{EJEMPLARES COLECTADOS (Fig. 3)}

Estado de México:

Mpio. Texcoco. San Nicolás Tlamincas, $6 \mathrm{~km}$ al E de Texcoco. En terrenos de pastoreo, parte posterior de la iglesia. 2400 m. 27 septiembre 1992. J. García P. s. n. (IEB).

Mpio. Texcoco. Montecillo. Terrenos del Colegio de Postgraduados en Ciencias Agrícolas, cerca del edificio de Botánica. Terreno perturbado. Único ejemplar. 19²7'45" N, 9854'21" W, 2240 m. Mayo 1998. H. Vibrans s. n. (IEB).

Mpio. Aculco. Autopista México - Querétaro. Tierra suelta en la orilla de la carretera. Único ejemplar floreciendo; los demás (aproximadamente 50 ) ya están secos. $20^{\circ} 08^{\prime} 55.2^{\prime \prime} \mathrm{N}$, 9944'13.6" W, 2536 m. 23 noviembre 2000. H. Vibrans 6965. (CHAPA, IEB, MEXU).

Mpio. Texcoco, Cabecera municipal. Prolongación Av. 16 de Septiembre, a $100 \mathrm{~m}$ del entronque con la carretera Texcoco - Apizaco - Veracruz. Terreno baldío con vegetación esparcida y ruderal. Cinco individuos. 19³1'17.4" N, 9852'45.2" W, 2265 m s.n.m. 12 mayo 2001. H. Vibrans 7172 (CHAPA, IEB, MEXU).

Mpio. Lerma. Carretera México - Toluca, entre Lerma y Metepec (Paseo Tollocan), a la altura de Sta. Elena. Vegetación ruderal en camellón central de autopista. Población densa de aproximadamente $10 \mathrm{~m}^{2}$. 19¹7'05.3" N, 99³1'59.4" W, 2590 m s.n.m. 10 junio 2001. H. Vibrans 7295 (CHAPA, IEB, MEXU).

Mpio. Ixtapaluca. Carretera libre México - Puebla, Colonia Ávila Camacho, dentro del poblado. Vegetación ruderal a orilla de camino. Población grande y al parecer bien 


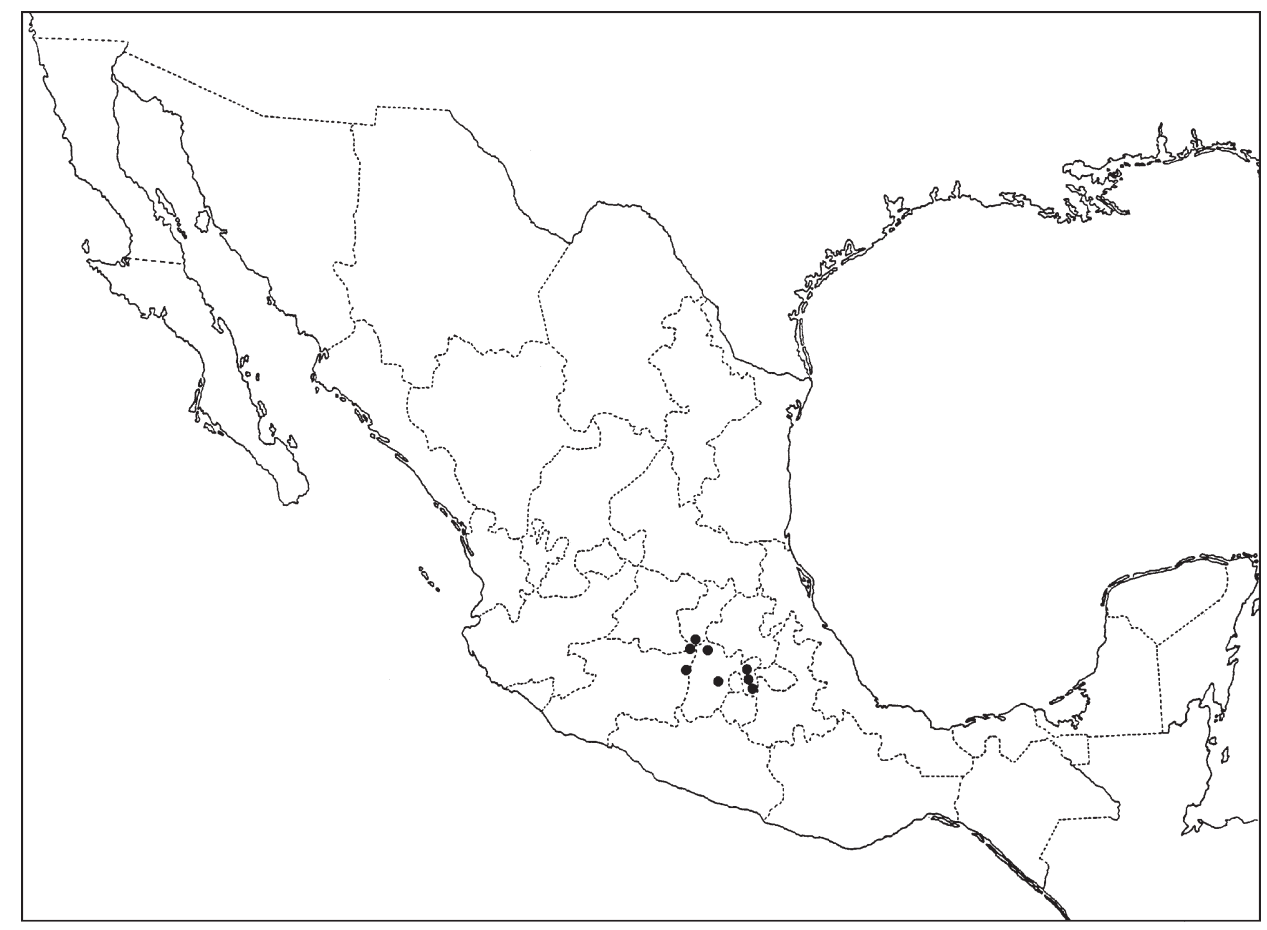

Fig. 3 Mapa de la República Mexicana en el cual se señalan las localidades conocidas de Senecio inaequidens.

establecida. 19¹9'22.9" N, 9845'41.1" W, 2910 m s.n.m. 16 julio 2001. H. Vibrans 7388 (CHAPA, IEB, MEXU).

Michoacán:

Mpio. Contepec. $3 \mathrm{~km}$ al este de Puerto Medina, próximo a Zaragoza. Orilla de la vía de ferrocarril. Planta escasa. 2550 m. 6 mayo 1997. J. Rzedowski 53348 (IEB).

Mpio. Epitacio Huerta. La Cima. Pastizal con restos de bosque de encinos, en laderas de pendiente suave, abundante. 2700 m. 27 agosto 2001. S. Zamudio 11806 (IEB). Cerca de La Cima. Bosque perturbado de encino; escasa en lugares perturbados. 2600 m. 20 octubre 2001. J. Rzedowski 53851 (IEB). 
Rzedowski et al.: Senecio inaequidens, una maleza perjudicial introducida en México

Querétaro:

Mpio. Amealco. $1 \mathrm{~km}$ al $\mathrm{W}$ de Amealco, sobre la carretera a Coroneo. Orilla de camino. 2650 m. 14 octubre 1990. J. Rzedowski 50324 (IEB).

Mpio. Amealco. El Picacho, desviación a Sn. Pedro Tenango a $3 \mathrm{~km}$ al SE de Amealco. Bosque de encino. 2650 m. "manzanilla de llano". 23 octubre 1999. V. Serrano 720 (IEB).

Mpio. Amealco. Amealco, alrededores de la población. Orilla de camino; muy abundante. 2650 m. 23 junio 2001. J. Rzedowski 53781 (IEB).

\section{AGRADECIMIENTOS}

Constituye un deber el reconocimiento de la ayuda recibida de diferentes personas para la cabal integración de este trabajo.

El Sr. José García-Pérez fue uno de los primeros en colectar y el primero en llamar la atención sobre la distinción de Senecio inaequidens de las especies mexicanas conocidas de este género.

La Dra. Rosaura Grether, de la Universidad Autónoma Metropolitana, comparó detalladamente los materiales mexicanos con los existentes en los herbarios de Australia y proporcionó copias de importantes trabajos realizados en aquel país.

La Dra. Lourdes Rico, de los Reales Jardines Botánicos de Kew, proporcionó fotografías de ejemplares de herbario y de literatura disponible en su institución.

Las excelentes fotografías de la planta son obra del Biól. Pedro Tenorio.

El Dr. Jean-Noël Labat, del Museo Nacional de Historia Natural de París, el Dr. Carlos Montaña, del Instituto de Ecología, A.C., el Bibl. Armando Butanda, del Instituto de Biología de la Universidad Nacional Autónoma de México, y el Dr. Gerwig Vibrans, de Berklingen, Alemania, facilitaron asimismo copias de publicaciones relevantes.

El Prof. C. G. Aymonin, del Museo Nacional de Historia Natural de París, tuvo la amabilidad de conectarnos con algunos botánicos franceses, activos en la actualidad en la problemática de la apropiada identificación y delimitación de Senecio inaequidens.

La Dra. Lucile Lafuma, de la Universidad de Montpellier 2, envió información sobre los avances de sus investigaciones citológicas y proporcionó una lista bibliográfica de la mayor trascendencia.

El Dr. Victor W. Steinmann, del Instituto de Ecología, A.C. tuvo la gentileza de revisar y corregir substancialmente el resumen en inglés.

\section{LITERATURA CITADA}

Alonso, S. I., O. N. Fernández, S. I. Langero y C. A. Verona. 1982. Characteristics of seed germination of Senecio madagascariensis Poiret (Compositae). Ecología Argentina 7: 95-116. (El artículo está escrito en español).

Böhmer, H. J. 2001. Das schmalblättrige Greiskraut (Senecio inaequidens DC. 1837) in Deutschland - eine aktuelle Bestandsaufnahme. Floristische Rundbriefe 35(1/2): 47-54. 
Cabrera, A. L. 1963. Senecio. In: Flora de la provincia de Buenos Aires. Colección Científica del I.N.T.A. Buenos Aires. pp. 282-319.

Cabrera, A. L. y R. R. Ré. 1965. Sobre un Senecio adventicio en la provincia de Buenos Aires. Revista de la Facultad de Agronomía de la Universidad Nacional de La Plata 41: 43-50.

Ernst, W. H. O. 1998. Invasion, dispersal and ecology of the South African neophyte Senecio inaequidens in The Netherlands: from wool alien to railway and road alien. Acta Botanica Neerlandica 47: 131-151.

Fernández, O. N. y C. A. Verona. 1984. Características reproductivas de Senecio madagascariensis Poiret (Compositae). Revista de la Facultad de Agronomía de la Universidad de Buenos Aires 5: $125-137$.

García-Pérez, J., 2001. Senecio. In: C. de Rzedowski, G., J. Rzedowski y colaboradores. Flora fanerogámica del Valle de México, 2a. ed. Instituto de Ecología, A.C. y Comisión Nacional para el Conocimiento y Uso de la Biodiversidad. Pátzcuaro, Mich. pp. 933-949.

Harden, G. J. 1992. Senecio. In: Harden, G. J. (ed.). Flora of New South Wales. New South Wales University Press. Kensington. Vol. 3. pp. 299-312.

Hilliard, O. M. 1977. Compositae in Natal. University of Natal Press. Pietermaritzburg.

Kinoshita, S., H. Koyama, M. Ogawa y O. Michihito. 1999. Senecio madagascariensis, a naturalized species in Japan. Acta Phytotaxonomica et Geobotanica 50: 243-246.

Kuhbier, H. y H. E. Weber. 2003. Senecio inaequidens als Bestandteil der natürlichen Dünenvegetation auf den Ostfriesischen Inseln. Tuexenia 23: 367-371.

Mandak, B. y K. Bimova, 2001. Novy druh jihoafrickeho starcku v Ceske republice: Senecio inaequidens. Zprávy Ceské Botanické Spoleđnosti (Praga) 36: 29-36.

Motooka, P., G. Nagai, K. Onuma, M. DuPonte, A. Kawabata y G. Fukumoto. 1999. Control of fireweed (Senecio madagascariensis). College of Tropical Agriculture and Human Resources Publication on Weed Control WC-2. University of Hawaii, Manoa, Hawaii. Disponible en: www2.ctahr.hawaii.edu/oc/freepubs/pdf/WC-2.pdf.

Pignatti, S. 1982. Senecio. In: Flora d'Italia. Edagricole. Bologna. Vol. 3. pp. 116-136.

Polatschek, A. 1984. Senecio inaequidens DC. neu für Österreich und Spanien. Verhandlungen der Zoologisch-Botanischer Gesellschaft in Wien 122: 93-95.

Radford, I. J. y R. D. Cousens, 2000. Invasiveness and comparative life-history traits of exotic and indigenous Senecio species in Australia. Oecologia 125: 531-542.

Scott, L. J., B. C. Congdon y J. Playford. 1998. Molecular evidence that fireweed (Senecio madagascariensis, Asteraceae) is of South African origin. Plant Systematics and Evolution 213: 251-257.

Sindel, B. M., 1996. Impact, ecology and control of the weed Senecio madagascariensis in Australia. In: Caligari, P. D. S. y D. J. N. Hind (eds.). Compositae: biology and utilization. Proceedings of the International Compositae Conference, Kew, 1994. Royal Botanic Gardens, Kew. Vol. 2. pp. 339-349.

Verona, C. A., O. N. Fernández, L. Montés y S. I. Alonso, 1982. Problemática agroecológica y biología de Senecio madagascariensis Poiret (Compositae). I. Problemática agroecológica y biología de la maleza. Ecología Argentina 7: 17-30. 